\title{
Activismo estudiantil, investigación y extensión universitaria: posibilidades en torno a la accesibilidad
}

\author{
Student activism, research and college extension: Accessibility \\ possibilities
}

Palabras clave

Accesibilidad, discapacidad, inclusión, activismo.

\section{Keywords}

Accessibility, disability, inclusion, activism.

\section{Lelia Schewe}

$<$ lelia.schewe@gmail.com>

Consejo Nacional de Investigaciones Científicas y Técnicas de Argentina (CONICET). Argentina

\section{Primeros pasos y "junturas"}

Este trabajo pretende narrar algunas experiencias vinculadas a la investigación y la extensión en una universidad del norte de Argentina, a partir de un espacio común que articula varios proyectos formalizados institucionalmente y un espacio de militancia estudiantil. Las dinámicas de participación en las universidades que no cuentan con recursos específicos para la accesibilidad se construyen de formas particulares, con aportes voluntarios de quienes creen que es posible y necesario lograr la participación de todos los estudiantes.

Después de haber investigado cuestiones normativas y legales con respecto a la inclusión de estudiantes pertenecientes a minorías sociales ${ }^{1}$ y específicamente, sobre las formas que adquiría ese concepto en el caso del trabajo universitario con estudiantes con discapacidad ${ }^{2}$, decidimos iniciar un recorri-

1. Proyecto "Inclusión de indígenas, personas portadoras de discapacidad, inmigrantes y de los sectores populares en la universidad" (PIUNI-Código 16H454). Se desarrolló durante los años 20172020. Entre los objetivos planteamos: 1. Relevar y describir políticas públicas y programas que promuevan la inclusión de estudiantes indígenas, personas portadoras de discapacidad, inmigrantes y de los sectores populares en la UNaM, 2. Describir y analizar las concepciones sobre la inclusión de los sujetos ya definidos, que tienen diversos integrantes de la comunidad universitaria y 3 . Identificar, describir y analizar las perspectivas de los estudiantes de las poblaciones seleccionadas acerca de sus experiencias de inclusión.

2. Proyecto "Inclusión de estudiantes portadores de discapacidad en la universidad (PIEDU- 16H509PI)". Se desarrolló entre 2018 y 2019. Los objetivos se escriben más adelante. Ambos proyectos se desarrollan en la Universidad Nacional de Misiones, Argentina.

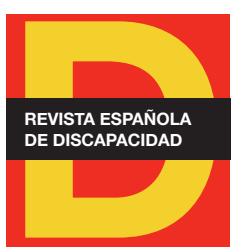

Para citar:

Schewe, L. (2020). "Activismo estudiantil, investigación y extensión universitaria: posibilidades en torno a la accesibilidad". Revista Española de Discapacidad, 8(2), pp. 255-263.

Doi: <https://doi.org/10.5569/23405104.08.02.14> 
do que implicara, desde las narrativas, a los protagonistas de estos procesos: comenzamos a trabajar construyendo biografías de estudiantes con discapacidad que actualmente están cursando estudios en la universidad.

En ese camino, nos encontramos con un interés común: era necesario crear un movimiento que pudiera sostener acciones de reivindicación de aquellos derechos que estaban siendo vulnerados. Como sucede en espacios que no son tan extensos y masivos, investigadores y participantes nos conocíamos, nos encontrábamos en las clases y habíamos leído producciones de los demás: lo único que no habíamos hecho, era juntarnos.

Así que, una tarde decidimos encontrarnos a orillas de un río, fuera del espacio de la universidad, en una gran ronda: estudiantes con discapacidad e integrantes de equipos de investigación, que hasta ese día habíamos estado distinguiéndonos así. A partir de ese encuentro, comenzamos a ser un equipo, tejiendo lo que luego se convertiría en el "Continuar: proyecto de accesibilidad universitaria". Coincidimos, inicialmente, en que necesitábamos discutir sobre las barreras físicas y las barreras actitudinales.

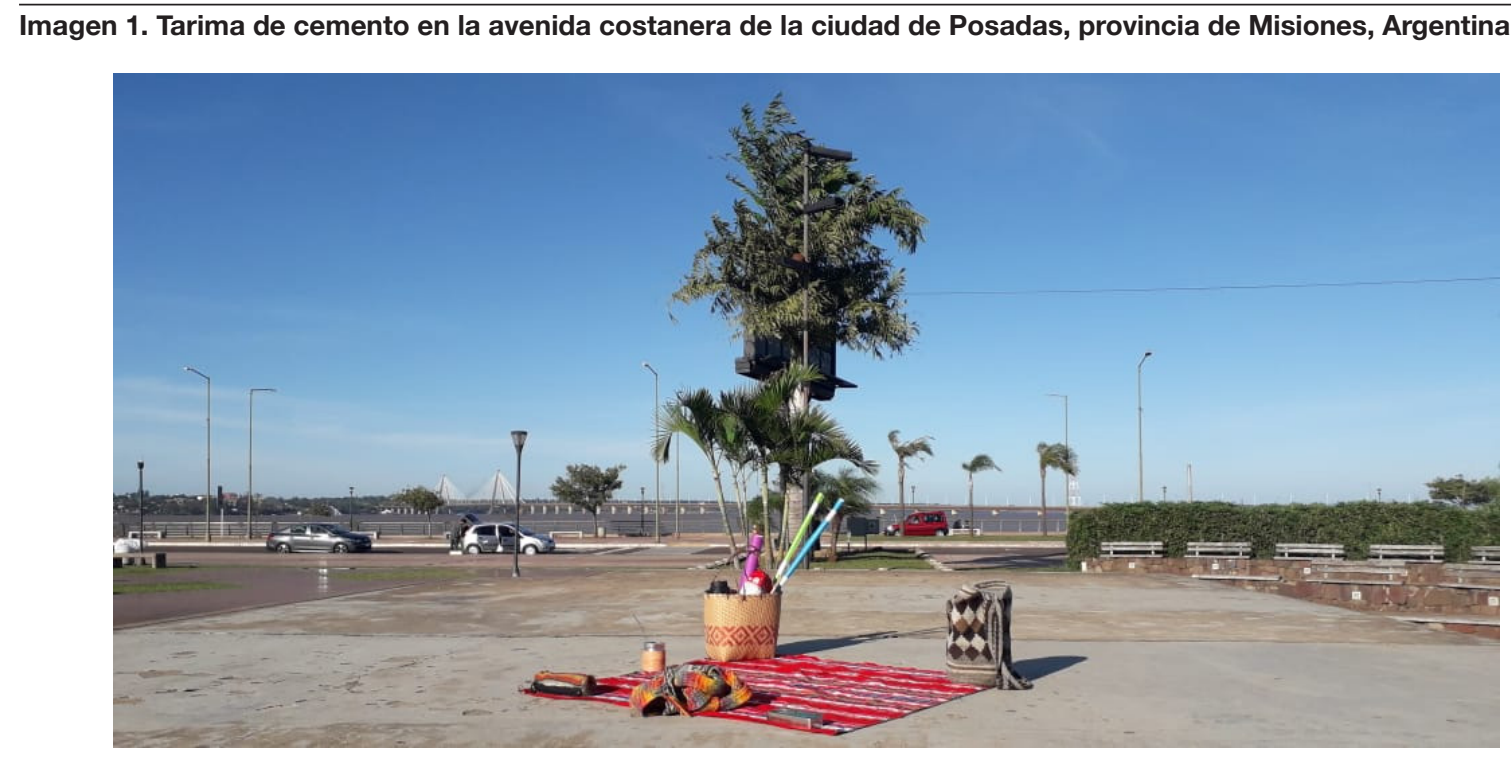

El grupo de militancia estudiantil que se había conformado llevó inicialmente el nombre de "Movimiento de estudiantes por la inclusión". La apertura que implicó nombrarse de esa manera permitió que se sumaran otros colectivos con intenciones de accionar para que la universidad contemplara su condición, necesidades y solicitudes, para que puedan continuar estudiando. Más tarde, las discusiones en torno al concepto de inclusión, las múltiples formas de identificarse con algún colectivo y las posiciones que manifestaban que no era necesario "ser incluidos" sino, solicitar o exigir apoyos, llevaron a que el nuevo nombre sea: "Movimiento de Estudiantes en Unidad".

En esos mismos momentos iniciales, se organizaron unas actividades que permitieron dos discusiones fundamentales: un foro de pueblos indígenas en la universidad, donde discutimos que otra de las barreras se 
corresponde con una cuestión cultural y un congreso de Educación Especial, que demostró con claridad la existencia de barreras comunicacionales.

\section{2. "Continuar"}

El proyecto de extensión tomó ese nombre porque nos interesa el trabajo con aquellos estudiantes que pudieron ingresar en la universidad, pero que no están seguros de que los apoyos que reciben y las condiciones para sus estudios sean suficientes. Nos habíamos encontrado, en los espacios de investigación ya mencionados, con la explicitación de dos cuestiones que consideramos centrales:

1. Existe una multiplicidad de formas de interpretar los planteamientos de las políticas públicas que promueven la inclusión de estudiantes que requieren apoyos en la educación superior.

2. Las limitaciones contextuales con las que se enfrentan cotidianamente los integrantes de estos colectivos en los espacios universitarios pueden implicar interrupciones y abandono de sus carreras universitarias.

Las configuraciones de apoyo (Pereyra, 2017) para el acceso a los espacios de clase dependen de los recursos con los que cuente cada estudiante o de las voluntades individuales de los profesores. Coincidimos con Seda (2014) en que las instituciones universitarias presentan una contradicción con el mensaje de apertura irrestricta y la deuda de apoyos suficientes para estudiantes que los necesitan para acceder a la educación superior. Según los informes de avance de los proyectos de investigación que mencionamos (PIUNI y PIEDU), los estudiantes expresaron que la falta de cuestiones mínimas como los subtítulos en un material audiovisual o el formato de presentación de los textos de las asignaturas universitarias, puede significar la interrupción de las trayectorias académicas e inclusive, el abandono. En este caso, si bien se trata de prácticas que podrían resultar sencillas de resolver, la suma de todas las vicisitudes cotidianas les demanda una gran cantidad de tiempo y esfuerzo extra.

Por otra parte, explicitaron haber tomado medidas extremas, como levantar en brazos a una compañera con una discapacidad motriz para que participe en clases en un tercer piso, debido al mal funcionamiento de un ascensor y a que las aulas de la planta baja de un edificio se encontraban ocupadas; esto quiere decir que, para los estudiantes con discapacidad motriz aparecen espontáneamente por este tipo de cotidianeidades, barreras arquitectónicas que se suman a otras, ya existentes. En el caso de los estudiantes con discapacidad visual, los laboratorios de informática y las bibliotecas se vuelven lugares inaccesibles, debido a la ausencia de material bibliográfico en sistema Braille y a la falta de lectores de pantallas en las computadoras. Los estudiantes de pueblos indígenas han manifestado ser víctimas de múltiples situaciones de discriminación por el desconocimiento - por parte de quienes forman parte de la gestión y el trabajo cotidiano en la universidad - acerca de la cultura y problemáticas por las que atraviesan sus pueblos, como afirma Núñez (2017). Manifestaron dificultades en su ingreso y tránsito por el sistema educativo superior porque el castellano representa su segunda lengua, lo que afecta directamente la lectura y comprensión de los textos académicos. 
Los estudiantes cuyas identificaciones se construyen desde las formas no binarias del género, narran las maneras en que los espacios universitarios responden a la heteronormatividad hegemónica (Morgade, 2016), porque no se contemplan otras posibilidades de ser en las propuestas curriculares. Las situaciones de violencias vinculadas a los géneros, especialmente contra las mujeres, también son una preocupación en nuestro contexto: actualmente se conforman grupos de contención y lucha contra este flagelo.

Entonces, las formas que adquieren las barreras que mencionamos, serían:

- Barreras culturales: invisibilización, infantilización, desconocimiento.

- Barreras actitudinales: inferiorización, alejamiento y exclusión, discriminación.

- Barreras edilicias: falta de señalética en Braille e iluminación acorde, falta de alertas y mensajes sonoros, arquitectónicas.

- Barreras curriculares: materiales no accesibles, páginas Web no operables, falta de intérpretes de lengua de señas argentina (LSA).

Para formalizar institucionalmente el proyecto, lo presentamos a una convocatoria para programas de extensión (Programa de Fortalecimiento de las Actividades de Extensión-PROFAE) de la Secretaría General de Extensión Universitaria y obtuvimos el primer puesto en el rubro de trabajos interinstitucionales. Al momento de la formalización, contábamos con la participación de veintidós integrantes, entre estudiantes, docentes y personal administrativo, pertenecientes a cuatro unidades académicas de la universidad.

\section{Algunas respuestas}

Iniciamos las actividades formalmente proponiéndonos un plan de trabajo extenso, para un año de duración. Partimos de los desarrollos actuales disponibles sobre educación superior en Argentina, en torno a tres temáticas, propuestas a priori y modificables según los avances del proyecto:

- Estudiantes con discapacidad y configuraciones de apoyo en la educación superior.

- Estudiantes de comunidades indígenas y accesibilidad académica.

- Disidencias sexuales e identificaciones "otras" en la universidad.

Las estrategias que planificamos corresponden a tres etapas de trabajo, necesarias desde un diagnóstico inicial, porque no hay datos disponibles sobre las poblaciones que identificamos:

1. Estrategias diagnósticas. A partir de visitas a las unidades académicas, nos propusimos determinar y registrar cuáles son las condiciones de accesibilidad para aquellos estudiantes que necesiten apoyos. También fue fundamental, analizar la pertinencia de los apoyos existentes, tomando como referencia los estudiantes que cursan carreras en cada facultad, sistematizando, además, las barreras actuales para el acceso de todos los estudiantes. Por otro lado, planificamos la realización de encuestas a los estudiantes y reuniones con los equipos de gestión en cada facultad, para conocer las opiniones sobre 
la accesibilidad. Toda esta información está siendo sistematizada a los fines de generar una base de datos que se encontrará disponible para futuras acciones vinculadas.

2. Estrategias de concientización. Creemos que realizar charlas en cada unidad académica, a partir de los diagnósticos, sería interesante para comenzar a generar debates críticos. Se propuso un coloquio sobre accesibilidad en la educación superior, invitando a referentes de otras universidades que se encuentren trabajando la temática y desarrollando proyectos de intervención. Además, la realización de radios abiertas y un ciclo radial que incluye aspectos como: espacios laborales para personas con discapacidad, trayectorias escolares, educación sexual integral, formación docente e inclusión, políticas públicas.

3. Estrategias de intervención directa. Como ejemplos, mencionamos el diseño y la colocación de señalética en Braille y mapas hápticos a la entrada de cada edificio, la solicitud de intérpretes de lengua de señas argentina (LSA), la traducción de textos al idioma guaraní ${ }^{3}$, entrega de folletos a docentes y gestores de espacios académicos sobre disidencias sexuales e identidades no binarias. En esta etapa nos encontramos trabajando actualmente, con los desafíos que implica hacerlo en tiempos de COVID 19.

\section{Conversatorio sobre feminismos con perspectiva de discapacidad}

Lucía, Betzabé y Belén son estudiantes militantes feministas. La preocupación —basada en su propia experiencia - por las formas en que se excluye a las mujeres con discapacidad de ciertos espacios, como las clases de Educación Sexual Integral (ESI), les condujo a proponer el conversatorio, en el marco del "Continuar". Compartimos algunas preguntas que plantearon: ¿suponen que es fácil imaginar a qué se refieren cuando dicen "útero" y una tiene discapacidad visual? ¿Por qué no hay materiales disponibles en Braille sobre anticoncepción? ¿Saben que a las mujeres con discapacidad auditiva los ginecólogos las tocan sin explicarles qué les van a hacer? ¿Por qué a las mujeres con discapacidades motrices se les niegan los espacios de disfrute sexual?

Relataron sobre los espacios de resistencia de mujeres con discapacidad que son víctimas de violencias, y los grupos que están trabajando para volver accesible aquello que les está siendo negado. Sus voces se acercan a lo que plantean Mello y Mozzi (2018) cuando narran que los gestores del Modelo Social de la Discapacidad no refieren al dolor y los cuidados, como cuestiones políticas fundamentales en la vida de las mujeres, por lo que existe una necesidad de abrazar la interseccionalidad, desde las perspectivas feministas y la teoría crip.

Una participante del conversatorio, madre de una mujer con discapacidad, agradeció la oportunidad de estar, porque ya está cansada de especialistas soberbios que hablan sobre la discapacidad desde sus consultorios y los atriles, pero, como no la viven, la "miran desde afuera" y opinan. Se planteó que, cuando enseña cosas a su hija, no le pregunta sobre cómo percibe lo que se le enseña y que ella misma había tenido sospechas sobre las capacidades y posibilidades. Le resultó tranquilizador que haya mujeres con discapacidad, ocupándose de esos temas en las universidades.

3. Comunidad indígena originaria de la provincia de Misiones y zonas aledañas. 


\section{La radio abierta}

En un contexto de lucha docente, con represiones violentas a las movilizaciones que pedían salarios acordes al trabajo ${ }^{4}$, se realizó una radio abierta, como gesto de solidaridad, desde nuestro espacio universitario. La programación consistió en relatos sobre las condiciones de trabajo, la precarización, la criminalización de la protesta y el capacitismo.

Como en nuestro contexto no se visibilizan organizaciones y espacios de formación política que contemplen situaciones de discapacidad y pueblos indígenas, la instalación de la radio abierta, al frente de una de las sedes de la universidad, despertó varias reacciones, entre ellas, en el momento en que una estudiante estaba cantando, se escucharon gritos de burlas, provenientes de una de las oficinas del personal no docente de la sede.

Una de las cuestiones más interesantes del espacio radial, consistió en la presencia de un estudiante -que no había estado vinculado al proyecto - quien manifestó la necesidad de empezar a hablar sobre esos temas, que eran muy relevantes, hablando de la discapacidad como un asunto "de ellos". Como él coincidía visiblemente con lo que conocemos los demás como una discapacidad motriz, las discusiones sobre cómo nos cuesta no imponer categorías, aunque trabajemos en esas temáticas cotidianamente, fue propicia para discusiones que implican varias perspectivas sobre las formas de nombrar y las construcciones teóricas que volvimos a revistar al respecto:

- La perspectiva sociológica de la discapacidad (Oliver, 1998; Barton, 2008; Ferreira, 200; Ferrante, 2014).

- La perspectiva interseccional de la discapacidad (Serra, 2017; Munévar, 2012).

- La perspectiva feminista de la discapacidad (Morris, 2008; Mello, 2016; Viñuela, 2009; Moscoso, 2007; Balza, 2011).

- La perspectiva de los estudios críticos en la discapacidad en Latinoamérica y los estudios decoloniales sobre discapacidad (Morán y Tiseyra, 2019; Mancebo, 2015; Cruz et al., 2019; Barrozo et al, 2017; Rojas, 2015; Yarza de los Ríos et al., 2019).

\section{En tiempos de COVID-19}

El aislamiento social preventivo y obligatorio, demandó la reorganización de las actividades. Una de las más postergadas, por las urgencias de la cotidianeidad, había sido la de juntarnos a estudiar, así que fue el momento propicio para crear un espacio a esos fines, que nombramos: Semillero de Estudios Críticos en Discapacidad (SECRID).

4. Más información: http://revistaanfibia.com/ensayo/aula-esta-demasiado-vacia/. 
Cuando relevamos las posibilidades del trabajo virtual, nos encontramos con que el único medio posible para realizar foros (porque lo usan todos los integrantes del equipo) es la aplicación Whatsapp. Por eso, elegimos comenzar por ese medio, sobre las perspectivas de autoras mujeres latinoamericanas con discapacidad, que cuestionan críticamente las miradas colonizantes que han permitido violencias y exclusiones. Los intercambios están siendo interesantes, sobre todo porque los integrantes del grupo estudian y trabajan en campos disciplinares diferentes: historia, comunicación, antropología, educación especial, sociología y hasta matemáticas. Los encuentros virtuales resultan accesibles para todos, en este caso, también nos referimos a las posibilidades económicas de disponer de computadoras, acceso a internet, etc.

En tiempos de aislamiento por COVID-19, también fue necesario encontrarnos desde otros lugares. Creamos una red de sororidad feminista virtual, para contener a aquellas mujeres que son víctimas de violencias, son usuarias de terapias que no están disponibles en tiempos del COVID, están solas, tienen dificultades económicas, entre otras situaciones.

También participamos aportando a un relevamiento realizado por el principal organismo público de ciencia y tecnología de nuestro país, aportando datos sobre las situaciones de los colectivos que podrían encontrarse vulnerables por las nuevas dinámicas a partir de la pandemia.

Otra de las actividades pendientes, es la escritura sobre los temas que nos fueron quedando sin registrar y analizar, en estas complejidades. Entonces, se van esbozando textos sobre la primera experiencia de voto accesible en Braille de nuestra universidad, la participación política de las compañeras con discapacidad visual en un parlamento municipal y uno de los hitos históricos más relevantes para nuestro país, sobre la discapacidad: el Frente de Lisiados Peronistas de los años 70.

\section{Seguir tejiendo continuidades}

Urge decolonizar las miradas patriarcales, heteronormativas, racistas, como sostenemos desde las teorías feministas, pero también urge hacerlo sobre las miradas capacitistas, que definen un "estudiante universitario hegemónico".

Sabemos que no todos los estudiantes en determinadas situaciones necesitan las mismas cosas, reconocemos que el trabajo implica necesariamente conocer-nos en nuestras subjetividades y diferencias, también en las universidades. Una de las últimas discusiones interesantes que se suscitaron entre los docentes universitarios argentinos fue sobre la pertinencia de las clases virtuales, sabiendo que las desigualdades sociales son tan profundas que hay estudiantes que no tienen acceso a internet o no tienen teléfonos celulares.

Con esa conciencia necesaria, seguimos tejiendo continuidades. Vamos vislumbrando que la articulación entre militancia, investigación y extensión es posible, mientras luchamos cotidianamente para que las universidades sean espacios para todos y todas los y las estudiantes. 


\section{Referencias bibliográficas}

Balza, I. (2011). "Crítica feminista de la discapacidad: el monstruo como figura de la vulnerabilidad y exclusión". Dilemata, 7, pp. 57-76. Recuperado de https://dialnet.unirioja.es/servlet/articulo?codigo=3743414.

Barrozo, N. N. et al. (2017). "Trayectorias escolares de personas con discapacidad en la educación secundaria en Argentina”. En A. Rodríguez-Martín (comp.). Prácticas innovadoras inclusivas: retos y oportunidades (pp. 1595-1603). Oviedo: Universidad de Oviedo. Recuperado de http://digibuo.uniovi.es/dspace/bitstream/10651/50310/4/TrayectoriasEscolares.pdf.

Barton, L. (2008). Superar las barreras de la discapacidad. Madrid: Ediciones Morata.

Cruz, I. et al. (2019). Apropiación social del conocimiento en discapacidad: experiencias latinoamericanas. Bogotá: UDR.

Ferrante, C. (2014). "Usos, posibilidades y dificultades del modelo social de la discapacidad. Revista Inclusiones". Revista de Humanidades y Ciencias Sociales, 1(3), pp. 33-55.

Ferreira, M. A. V. (2005). "La reflexividad social transductiva: la construcción práctico-cognitiva de lo social y la sociología". Nómadas. Revista Crítica de Ciencias Sociales y Jurídicas, 11(1), pp. 287-303. Recuperado de https://revistas.ucm.es/index.php/NOMA/article/view/NOMA0505120287A.

Mancebo, M. (2015). ¿Puede el "discapacitado" como sujeto subalterno hablar? Breve estudio crítico sobre el accionar político-colectivo en torno a la discapacidad en Uruguay [Tesis de grado. Uruguay, Universidad de la República]. Recuperado de https://www.colibri.udelar.edu.uy/jspui/handle/20.500.12008/5201.

Mello, A. G. (2016). "Economia moral do cuidado: um estudo sobre violências contra mulheres com deficiência em Belo Horizonte". En A. M. Veiga et al. (org.), Gênero e Violências. Diálogos interdisciplinares (pp. 86-120). Florianópolis: Edições do Bosque.

Mello, A. G. y Mozzi, G. (2018). "A favor da deficiência nos estudos interseccionais de matriz feminista”. En M. V. de Freitas Rosa et al. (comps.). Políticas públicas, relações de gênero, diversidade sexual e raça na perspectiva interseccional (pp.17-30). Porto Alegre: Secco Editora.

Morán, J. A. P. y Tiseyra, M. V. (2019). "Encuentro entre la perspectiva decolonial y los estudios de la discapacidad". Revista Colombiana de Ciencias Sociales, 10(2), pp. 497-521.

Morgade, G. (2016). "Políticas de educación sexual integral-Saberes, prácticas y cuerpos en tensión". Retratos da Escola, 9(16), pp. 63-71.

Morris, J. (2008). "Lo personal y lo político: una perspectiva feminista sobre la investigación de la discapacidad física". En L. Barton (comp.). Superar las barreras de la discapacidad (pp. 315-326). Madrid: Morata.

Moscoso, M. (2007). "Menos que mujeres: los discursos normativos del cuerpo a través del feminismo y la discapacidad”. En J. Arpal e I. Mendiola (comps.). Estudios sobre cuerpo, cultura y tecnología (pp. 185-195). Servicio editorial de la Universidad del País Vasco. Recuperado de https://bit.ly/3mDTxKI.

Munévar, D. I. (2012). "Interseccionalidad y otras nociones”. En S. Galindo et al. (comps.). La interseccionalidad en debate (pp. 56-65). Berlín: MISEAL.

Núñez, Y. I. (2017). "Estudiantes Guaraníes, interculturalidad y educación superior en Misiones, Argentina”. Comparative Cultural Studies-European and Latin American Perspectives, 2(4), pp. 9-20. 
Oliver, M. (1998). “¿Una sociología de la discapacidad o una sociología discapacitada?”. En L. Barton (comp.). Discapacidad y Sociedad (pp. 34-58). Madrid: Morata.

Pereyra, C. (2017). "Las prácticas de las/os maestras/os de apoyo a la inclusión de un centro de servicios alternativos y complementarios". En A. Rodríguez Martín (comp.), Prácticas innovadoras inclusivas: retos y oportunidades (pp. 1103-1111). Oviedo: Servicio de publicaciones de la Universidad de Oviedo.

Rojas, S. (2015). "Discapacidad en clave decolonial. Una mirada de la diferencia". REALIS, 5(1), pp. 175-202. Recuperado de https://periodicos.ufpe.br/revistas/realis/article/viewFile/8836/8811.

Seda, J. (2014). "Acceder a la justicia para acceder a la salud.: La universidad y los derechos de las personas con discapacidad". +E: Revista de Extensión Universitaria, (4), pp. 44-48.

Serra, M. L. (2017). Mujeres con discapacidad: sobre la discriminación y opresión interseccional. Madrid: Midac.

Viñuela, L. (2009). "Mujeres con discapacidad: un reto para la teoría feminista". Feminismo/s, 13, pp. 33-48. Recuperado de http://rua.ua.es/dspace/handle/10045/13324.

Yarza de los Ríos, A. et al. (2019). Estudios críticos en discapacidad: una polifonía desde América Latina. Buenos Aires: CLACSO. 